\title{
Risk of acute stroke in patients with retinal artery occlusion
}

\author{
Sohan Singh Hayreh ${ }^{1}$
}

Received: 25 October 2019 / Accepted: 7 November 2019 / Published online: 25 November 2019

(c) The Royal College of Ophthalmologists 2019

\section{To the Editor:}

Based on meta-analysis, Fallico et al. [1] concluded "a prompt referral to the emergency department or stroke unit for neurological evaluation and brain imaging as part of the clinical management of acute retinal artery occlusion (RAO)". Over the years, I have seen about 500 patients with acute RAO. Based on that experience, I recently investigated at length the subject "Do patients with RAO need urgent neurological evaluation?" [2].

In the literature, when the association of RAO to stroke is discussed, RAO is almost invariably considered as if it was one disease, always caused by embolism. In fact, RAO comprises of central RAO, branch RAO, and cilioretinal artery occlusion and, unlike stroke, not all of them are always embolic in nature. For example, central RAO, although commonly embolic in nature, can also be due to a marked fall of perfusion pressure from a variety of causes other than embolism, or vasculitis. Branch RAO, similarly, although commonly embolic in nature, can also be due to vasculitis and other causes. The most common cause of cilioretinal artery occlusion is central retinal vein occlusion, and not embolism. As regards transient monocular vision loss (amaurosis fugax-AF), in my study of 2725 eyes with AF [3], only $12 \%$ had central RAO and $14 \%$ branch RAO. Transient ischemic attack (TIA) and AF are usually considered similar in nature. But TIA and AF are totally different clinical entities pathogenetically and clinically; TIA is cerebral in origin, whereas $\mathrm{AF}$ is ophthalmic in origin, and is not always due to transient retinal ischemia. Therefore, the assumption in the literature that all cases of RAO are due to retinal embolism, resulting in the association of RAO with stroke, has led to the perpetuation of misleading

Sohan Singh Hayreh

sohan-hayreh@uiowa.edu

1 University of Iowa, Iowa City, IA, USA information on the subject. Moreover, silent cerebral infarcts can develop without RAO.

Most importantly, both RAO and associated stroke/TIA are almost invariably due to a common denominator, i.e., embolism. From the practical point of view, therefore, the first essential for the management of RAO is to immediately find the source of the embolism causing the RAO (as well as, in some cases stroke/TIA), and then try to eradicate that source of embolism, if possible. This is much more urgent than undertaking a detailed, expensive neurological evaluation, unless neurological symptoms are present. What is needed is urgent out-patient evaluation, of the carotid artery, heart, fasting lipid levels and complete blood count, rather than in-patient urgent, expensive neurological evaluations. In view of that, I have never felt the necessity for those patients to undergo urgently a detailed neurological workup for stroke/TIA, unless, of course, there were neurological symptoms. On follow-up of a large cohort of RAO patients, I have found no negative results.

\section{Compliance with ethical standards}

Conflict of interest The author declares no conflicts of interest.

Publisher's note Springer Nature remains neutral with regard to jurisdictional claims in published maps and institutional affiliations.

\section{References}

1. Fallico M, Lotery AJ, Longo A, Avitabile T, Russo VA, Murabito P, et al. Risk of acute stroke in patients with retinal artery occlusion: a systematic review and meta-analysis. Eye (Lond). 2019. https://doi.org/10.1038/s41433-019-0576-y.

2. Hayreh SS. Do patients with retinal artery occlusion need urgent neurological evaluation? Am J Ophthalmol. 2018;196:53-56.

3. Hayreh SS, Zimmerman MB. Amaurosis fugax in ocular vascular occlusive disorders: prevalence and pathogeneses. Retina. 2014;34:115-22. 